\title{
Three-Phased Wake Vortex Decay
}

\author{
Fred H. Proctor ${ }^{*}$ and Nash'at N. Ahmad \\ NASA Langley Research Center, Hampton, Virginia 23681 \\ George S. Switzer \\ AS\&M, Inc., Hampton, Virginia 23666 \\ Fanny M. Limon Duparcmeur ${ }^{\S}$ \\ Eagle Aeronautics, Inc., Hampton, Virginia 23666
}

\begin{abstract}
A detailed parametric study is conducted that examines vortex decay within turbulent and stratified atmospheres. The study uses a large eddy simulation model to simulate the out-of-ground effect behavior of wake vortices due to their interaction with atmospheric turbulence and thermal stratification. This paper presents results from a parametric investigation and suggests improvements for existing fast-time wake prediction models. This paper also describes a three-phased decay for wake vortices. The third phase is characterized by a relatively slow rate of circulation decay, and is associated with the ringvortex stage that occurs following vortex linking. The three-phased decay is most prevalent for wakes imbedded within environments having low-turbulence and near-neutral stratification.
\end{abstract}

\section{Nomenclature}

wingspan of generating aircraft

initial vortex separation, $=\pi B / 4$

acceleration due to gravity, $=9.8 \mathrm{~m} \mathrm{~s}^{-2}$

wavenumber, $=2 \pi / \lambda$

Brunt-Vaisala frequency, $\quad=[(g / \theta) \partial \theta / \partial z]^{0.5}$

nondimensional Brunt-Vaisala frequency, $\quad=2 \pi N \mathrm{~b}_{0}{ }^{2} / \Gamma_{\mathrm{o}_{\odot}}$

radius from center of vortex

time coordinate ( $\mathrm{t}=0$ at vortex generation)

nondimensional time, $=t \mathrm{~V}_{\mathrm{o}} / \mathrm{b}_{\mathrm{o}}$

initial vortex descent velocity, $=\Gamma_{0} /\left(2 \pi b_{0}\right)$

along track coordinate

cross track coordinate

vertical coordinate

grid size in $x$-direction

pressure deviation from environmental pressure

nondimensional vortex circulation, $=\Gamma / \Gamma_{\mathrm{o}}$

initial vortex circulation

eddy (turbulence) dissipation rate

nondimensional eddy dissipation rate, $\quad=\left(\varepsilon b_{0}\right)^{1 / 3} V_{o}^{-1}$

component of vorticity in $x$ direction

potential temperature

wavelength

\footnotetext{
* Senior Research Scientist, Crew Systems \& Aviation Operations Branch, AIAA Senior Member.

${ }^{\dagger}$ Senior Research Scientist, Crew Systems \& Aviation Operations Branch, AIAA Senior Member.

* Senior Research Scientist, NASA Contractor, AIAA Senior Member.

${ }^{\S}$ Research Scientist, NASA Contractor.
} 


\section{Introduction}

A $\mathrm{S}$ an aircraft generates aerodynamic lift, vorticity is generated and shed by the aircraft's wings, flaps, tail and body. Within a few spans downstream, this vorticity rolls-up into a pair of counter-rotating vortices. These vortices extend downstream and may become a danger to following aircraft. The initial strength of the wake vortices is primarily dependent upon the generating aircraft's speed, weight, and wingspan, and to a lesser extent upon flight configuration and aircraft design. The wake vortices decay with time and become unrecognizable within about one-half to several minutes. Current aircraft separation rules are based on aircraft weight categories, and ensure safety by allowing enough separation so that wake vortices are not a danger for in-trail aircraft. Understanding and being able to predict the rate of decay of aircraft wake vortices is paramount in developing: wake separation standards for new aircraft, re-categorization of standards for current aircraft, and for investigating safety issues related to proposed concepts and procedures for aircraft traffic management (e.g., merging and spacing for closely spaced parallel approaches.)

There are several schools of thought regarding how wake vortices decay when they are out of ground effect (i.e., away from the ground's influence). One concept is that wake vortices undergo an enhanced level of decay following the time the vortices link and form crude vortex rings. Crow, ${ }^{1,2}$ Sarpkaya, ${ }^{3}$ and others have noted that the "linking time" may be related to the vortex lifetime and is dependent upon the intensity of ambient turbulence; i.e. stronger ambient turbulence results in earlier linking and shorter vortex lifetimes. Spalart ${ }^{4}$ suggests an alternative interpretation with the time of linking being stochastic. In his concept, little or no vortex decay occurs until the onset of vortex linking, at which time there is a collapse of circulation and a rapid destruction of the vortex. His concept is based upon theoretical arguments with guidance from direct numerical simulations of the incompressible Navier-Stokes equations. A two-phased level of decay has also been described by Holzapfel et al. ${ }^{5,6}$ and Proctor et al., ${ }^{7,8}$ with the initial decay (phase 1) being weak, followed by a rapid decay (phase 2) associated with the onset of vortex linking. These studies were based on large eddy simulations (LES), and showed that the rate of vortex decay (and lifetime) is a function of stratification as well as ambient turbulence. Furthermore, Proctor et al. found that the onset of vortex linking from Crow instability was independent of the background stratification; although increasing levels of stratification may lead to a faster demise due to the onset of small-scale instability.

Semi-empirical wake prediction models have been formulated from guidance provided by the LES. They are the Deterministic 2-Phased (D2P) model $^{6}$ and the TASS Driven Algorithm for Wake Prediction (TDAWP). ${ }^{8,9}$ Both D2P and TDAWP account for two-phased vortex decay. Other studies rooted in laboratory experiments, field data, and theory, have assumed a single-phased decay function for their predictions. The semi-empirical wake models of Greene $^{10}$ and Sarpkaya ${ }^{11}$ predict wake vortex decay as a function of atmospheric turbulence and stratification (Greene's model also contains a viscous drag term); but unlike the models developed from LES, they predict a rate of decay that is initially large and diminishes with time. This is in contrast to Holzapfel's D2P and Proctor et al.'s TDAWP, which have an initial phase of weak decay followed by an enhanced rate of decay (Figure 1). Lastly, linear decay models have been developed that are based on best fits to an ensemble of measurements that are taken over a range of weather conditions. ${ }^{12}$ Although linear models have an advantage of simplicity, theoretical justification for a linear circulation decay is weak when applied to aircraft wake vortices. Another shortcoming of linear models is that they are based on an ensemble fit of data from a field campaign; therefore, at best, they would represent the mean rate of decay as affected by the most common weather conditions.

The existence of wake vortex measurements has not resolved the controversies regarding wake vortex decay. Laboratory studies cannot obtain the high-Reynolds numbers needed to realistically simulate aircraft wake vortices. At low-to-moderate Reynolds numbers (as observed in the laboratory) wake vortices maintain a significant rate of early decay aided by the effect of molecular viscosity and core growth. Actual aircraft vortices have a Reynolds number on the order of $10^{7}$, and possess vortex cores that remain tight even as the vortex decays with time. In the field, lidar seems to be the most reliable sensor for measuring aircraft wake vortices at this time. A lidar senses the component of wind along the direction of the beam, and vortex circulation is then estimated by fitting a model profile to the wind measurement. Factors that can affect the accuracy of the measurements include: lidar pulse length, lidar processing assumptions, signal to noise ratio, geometry, range, atmospheric turbulence, atmospheric wind shear, and poor visibility. Currently, systematic studies are being sponsored by NASA that should quantify these errors and improve the lidar's ability to measure circulation strength. 

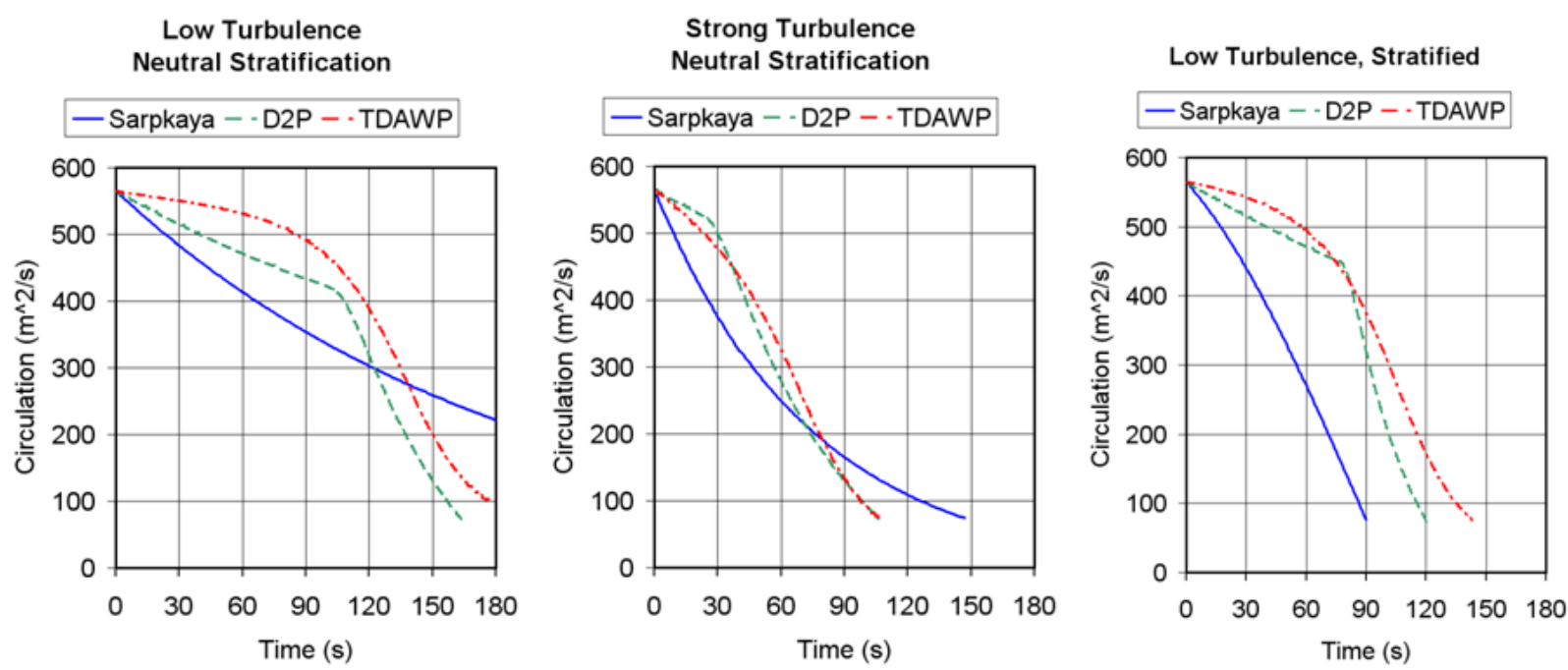

Figure 1. Circulation vs time comparison for fast-time out-of-ground-effect wake prediction models.

This paper examines recent results from wake vortex simulations under varying conditions of atmospheric turbulence and stability. The Terminal Area Simulation System ${ }^{13}$ (TASS) was used to perform the numerical study. In the following sections the TASS model is described briefly and the wake vortex simulations setup and results are discussed in detail.

\section{LES Model}

The TASS model has a meteorological framework and has initialization modules that allow the simulation of wake vortex interaction with the atmosphere. ${ }^{14,15,16}$ The model, being LES, explicitly resolves scales of turbulence larger than the grid size, while modeling subgrid scales via turbulence closure approximations. The subgrid turbulence formulation is dependent upon the local flow deformation, stratification, and flow rotation. TASS contains a compressible time-split formulation, and solutions for the equations of momentum are obtained with $4^{\text {th }}$ order quadratic-conservative space differences. The model is essentially free of numerical diffusion and is suitable for long-term integrations of vortex decay. ${ }^{17}$

\section{Initial Conditions}

This study examines the out-of-ground effect (OGE) behavior of a wake vortex pair following roll up; therefore, periodic boundary conditions are assumed. The initial vortex system consists of a pair of counter-rotating vortices that have no initial variation in the axial direction. The centers of each vortex are separated laterally by a distance $\mathrm{b}_{0}$. The initial vortex tangential velocity, $V$, is a function of radius, $r$, from the center of the vortex as ${ }^{18}$

$$
V(r)=\left(\Gamma_{\infty} / 2 \pi r\right)\left\{1-\exp \left[-10(r / B)^{0.75}\right]\right\}
$$

where $\Gamma_{\infty}$ is the vortex initial circulation. The dependency on wingspan $B$, rather than the vortex core radius $r_{c}$, is desirable since $B$ is easily determined from aircraft type while $r_{c}$ is difficult to accurately measure. The above equation is only applied for $r>1.4 r_{c}$. For $r \leq 1.4 r_{c}$, the model is matched with the Lamb model, ${ }^{19}$ i.e.

$$
V(r)=\left(\Gamma_{\infty} / 2 \pi r\right) 1.0939\left\{1-\exp \left[-10\left(1.4 r_{\mathrm{c}} / B\right)^{0.75}\right]\right\}\left\{1-\exp \left[-1.2527\left(r / r_{\mathrm{c}}\right)^{2}\right]\right\} .
$$

Simulations are conducted assuming aircraft parameters representing a B-747, as shown in Table 1. Specifications for the domain and grid size are shown in Table 2. The width and height of the domain is larger than in our earlier studies, ${ }^{14,15}$ in order to minimize boundary influences during the ring-vortex phase of evolution. 
Table 1. Initial vortex parameters for TASS simulations.

\begin{tabular}{|l|l|l|}
\hline Initial Vortex System Parameters & Symbol \\
\hline Circulation & $565 \mathrm{~m}^{2} \mathrm{~s}^{-1}$ & Гo \\
\hline Span & $63.66 \mathrm{~m}$ & $\mathrm{~B}$ \\
\hline Lateral separation & $50 \mathrm{~m}^{-1}$ & bo \\
\hline Descent speed & $1.8 \mathrm{~m} \mathrm{~s}^{-1}$ & $\mathrm{Vo}_{\mathrm{o}}$ \\
\hline Vortex core radius & $4.5 \mathrm{~m}$ & $\mathrm{r}_{\mathrm{c}}$ \\
\hline
\end{tabular}

Table 2. Domain size and resolution.

\begin{tabular}{|l|l|l|}
\hline Domain Parameters & Physical Dimension & Vortex Spacing Units \\
\hline Longitudinal dimension & $800 \mathrm{~m}$ & 16 bo \\
\hline Lateral and vertical dimensions & $600 \mathrm{~m}$ & 12 bo \\
\hline Longitudinal grid spacing & $2 \mathrm{~m}$ & $1 / 25 \mathrm{~b}_{\mathrm{o}}$ \\
\hline Lateral and vertical grid spacing & $1.5 \mathrm{~m}$ & $\approx 1 / 33 \mathrm{~b}_{\mathrm{o}}$ \\
\hline
\end{tabular}
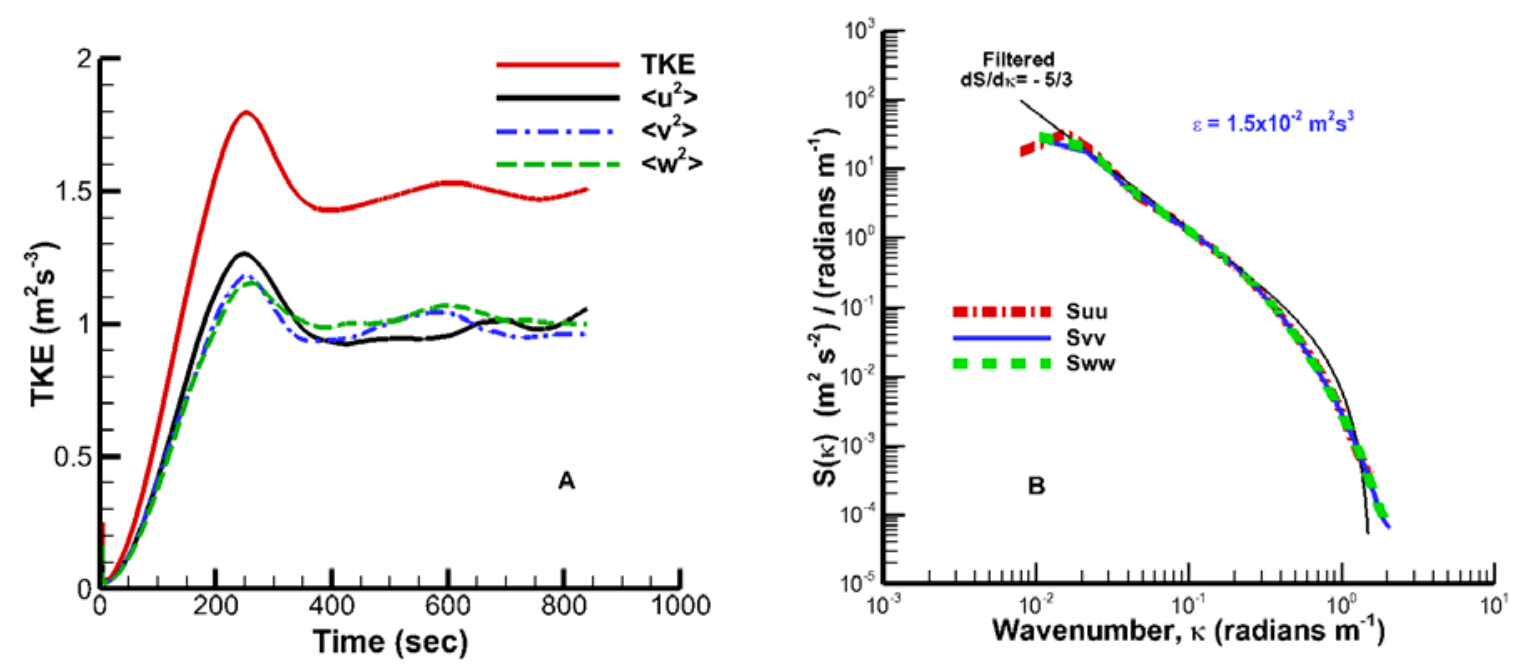

Figure 2. Turbulence statistics before vortex injection: a) time evolution of turbulence kinetic energy and velocity variance; $b$ ) three-dimensional spectrum of steady-state turbulence field.

Prior to vortex initialization, an initial field of resolved-scale turbulence is allowed to develop under an artificial external forcing at low wavenumbers. ${ }^{15,20,21}$ As shown in Figure 2, the turbulence field is allowed to develop until the turbulence statistics become nearly steady (about 18 eddy turn-over times).

The spectrum of the steady-state turbulence (Figure $2 b$ ) shows a well-defined inertial subrange and a $-5 / 3$ slope through most wavenumbers. A steepening of the slope near the cut-off wavenumber is expected since velocities predicted numerically by LES represent volumetric averages rather than unaveraged point values (see appendix). The turbulence kinetic energy dissipation rate is estimated by fitting Kolmogorov's ${ }^{22}$ theoretical spectrum to the 
simulated spectra. Once a turbulence field is grown, its velocity field can be rescaled to represent different turbulence intensities and used as an initial field for parametric wake vortex simulations. ${ }^{14}$

Thermal stratification is added to the parametric set of experiments by defining a vertical gradient of potential temperature. A specific value for $N^{*}$ is selected for an experiment and the potential temperature gradient is solved accordingly. In order to maintain isotropic and homogenous characteristics, the turbulence field is not allowed to respond to the thermal stratification prior to vortex injection. Other assumptions in this study include: no mean wind, a dry atmosphere, and no effects from jet blast.

Parameters for the study include a range of turbulence intensity between $\epsilon^{*}=0.05$ to 0.75 , and thermal stratification ranging between $N^{*}=0$ to 1.0 (Table 3). The matrix of 29 cases for this study has been considerably broadened from those performed in Switzer and Proctor $^{14}$ and which were used in the development of TDAWP. ${ }^{8}$

Table 3 Matrix of experiments

\begin{tabular}{|c|c|c|c|c|}
\hline \multirow{2}{*}{$\begin{array}{l}\text { Normalized } \\
\text { Turbulence } \\
\text { Intensity, } \text { c }^{*}\end{array}$} & \multicolumn{4}{|c|}{ Thermal Stratification, $\mathbf{N}^{*}$} \\
\hline & 0.0 & 0.25 & 0.75 & 1.0 \\
\hline .005 & $\mathbf{X}$ & $\mathbf{X}$ & $\mathbf{X}$ & $\mathbf{X}$ \\
\hline 0.03 & $\mathbf{X}$ & $\mathbf{X}$ & $\mathbf{X}$ & $\mathbf{X}$ \\
\hline 0.07 & $\mathbf{X}$ & $\bar{X}$ & $\mathbf{X}$ & $\mathbf{X}$ \\
\hline 0.15 & $\mathbf{X}$ & $\bar{X}$ & $\mathbf{X}$ & $\mathbf{X}$ \\
\hline 0.20 & $\mathbf{X}$ & $\mathbf{X}$ & $\mathbf{X}$ & $\mathbf{X}$ \\
\hline 0.25 & $\mathbf{X}$ & $X$ & $\mathbf{X}$ & \\
\hline 0.30 & $\mathbf{X}$ & $\bar{X}$ & & \\
\hline 0.35 & $\mathbf{X}$ & $\mathbf{X}$ & & \\
\hline 0.50 & $\mathbf{X}$ & & & \\
\hline 0.75 & $\mathbf{X}$ & & & \\
\hline
\end{tabular}

\section{Post-Processing of Results}

For each numerical experiment, TASS generates three-dimensional data fields at three-second intervals throughout the lifetime of the wake vortex. TASS results are post-processed using wake tracking software to compute the time evolution of vortex strength and trajectory. The wake tracking algorithm is described in this section.

Position and circulation for both the port and starboard vortex are computed for each cross-sectional $(y-z)$ plane of the computational domain. These cross-sectional $(y-z)$ plane values are used to compute the mean of the vortex circulation and position that are representative of the whole computational domain at a specific time interval. Special care is required in computing the mean values; especially, once the wake links to form ring vortices, or when strong turbulence and secondary vortices are present. For example, the connector filaments between the ring vortices (see Figure 3) can have weak circulations, and if included, can greatly reduce the mean circulation and increase the mean altitude. To avoid such errors, discriminators are defined which exclude a plane if certain thresholds are not met. Currently, the thresholds are applied on vorticity, pressure deviation, and the intersecting vortex angle with the cross-sectional plane. Recent improvements in the tracking algorithm allow for better diagnoses of vortex position and circulation, especially at later times of the vortex evolution.

The position $\left(y_{1}, z_{1}\right)$ of the wake vortices is computed for each cross-sectional plane according to:

$$
y_{1}=\frac{\iint y \Delta p^{2} \zeta^{3} d y d z}{\iint y d y d z}
$$


and,

$$
z_{1}=\frac{\iint z \Delta p^{2} \zeta^{3} d y d z}{\iint z d y d z}
$$

For calculation of the starboard vortex position, $\zeta$ is assumed to be zero at any grid point with $\zeta<0$, and vice versa for port vortex.

Circulation is computed for each cross-sectional plane with the above $\left(y_{1}, z_{1}\right)$ position representing the center of the vortex:

$$
\Gamma_{r}(x)=\iint_{r} \zeta \mathrm{d} y \mathrm{~d} z
$$

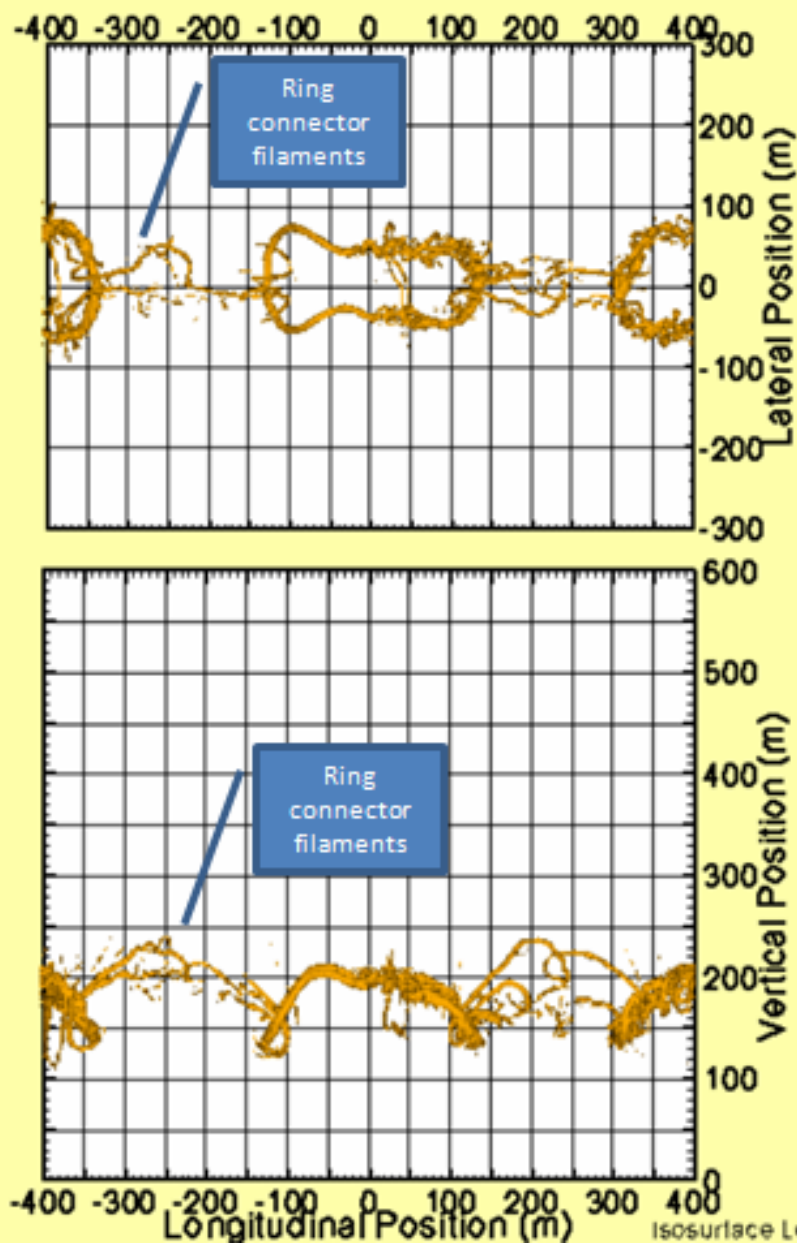

Figure 3. Top and side view of simulated wake vortex during ring vortex phase.

When applicable, the vortex linking time due to Crow (longwave) instability ${ }^{1}$ is determined for all 29 cases (Figure 4). As in our previous studies, we find that the linking time agrees with Sarpkaya's reanalysis ${ }^{24}$ of Crow and Bate's ${ }^{2}$ theory. In simulations with strong stratification, linking did not always occur due to the accelerated decay from short-wave ${ }^{25,26}$ instability. When vortex linking did occur, the linking time was found to be nearly unaffected by stable stratification $\left(N^{*}>0\right)$.

In previous studies with TASS, a two-phased decay was noted and incorporated into the TDAWP fast time model. ${ }^{8}$ Recent improvements in the post-processing analysis software, now allow better detection of circulation during the ring phase, and reveal a third phase as shown in Figure $5 \mathrm{a}$ and $5 \mathrm{~b}$.

As illustrated in Figure 5, wake decay can be broken into three stages. Phase 1 is represented by a relatively slow rate of decay and is followed by phase 2 with a more rapid rate of decay. Phase 2 is associated with the onset of vortex linking and $\mathrm{Crow}^{1}$ instability. A third phase, with a slower rate of decay follows and represents vortex decay during the vortex ring stage. This last stage of vortex decay is contrary to the concept of vortex collapse and sudden demise during the time of vortex linking. Laboratory studies support the findings that the decay rate is reduced during the ring vortex stage (D. Delisi, private communication). It should be noted, however, that an aircraft encounter with a ring vortex may be less severe than an encounter with a nearly two-dimensional vortex pair. ${ }^{27}$

A visualization of the wake vortex for each of the three phases of decay is shown in Figures 6-8. During phase-1, the vortex is nearly two-dimensional with minimal variation along its longitudinal direction. Linking occurs during phase 2 (Figure 7), and crudely shaped rings evolve by phase 3 (Figure 8). Our study shows that the vortex rings can persist long periods of time in conditions of very low atmospheric turbulence and neutral stratification. 


\section{Vortex Time to Linking vs Turbulence Intensity}

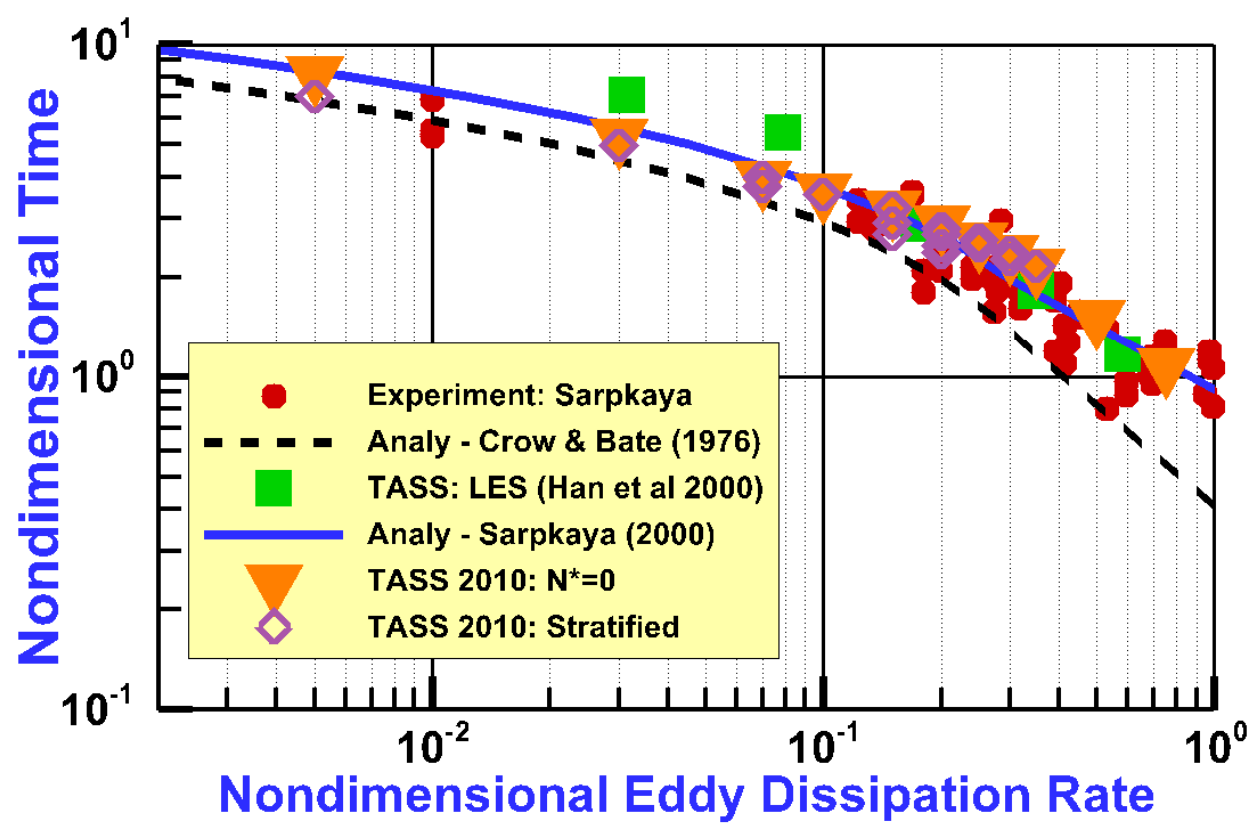

Figure 4. Wake vortex time to link vs. turbulence intensity. Present experiments represented by orange delta symbols (neutral stratification) and by purple diamond symbols $\left(N^{*} \geq 0.25\right)$.
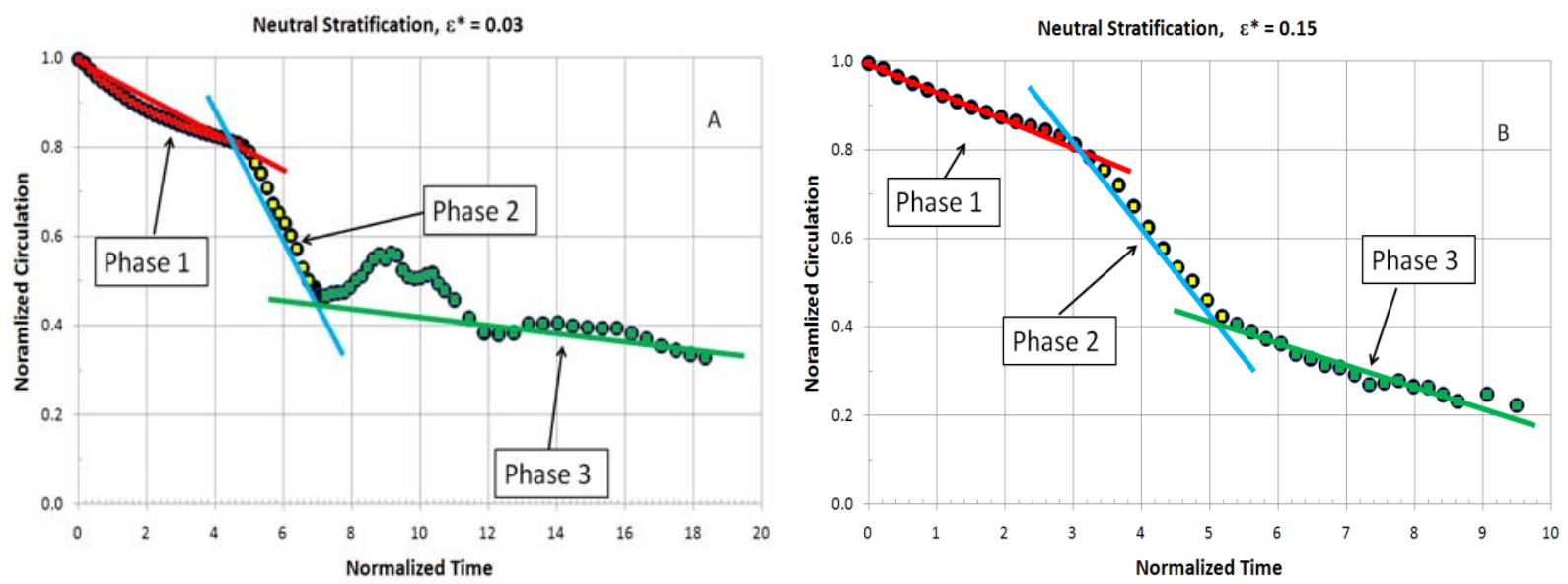

Figure 5. Nondimensional circulation vs. nondimensional time for two low-turbulence cases in a neutral atmosphere: a) $\cdot \varepsilon^{*}=0.03$, and b) $\cdot \varepsilon^{*}=0.15$. 


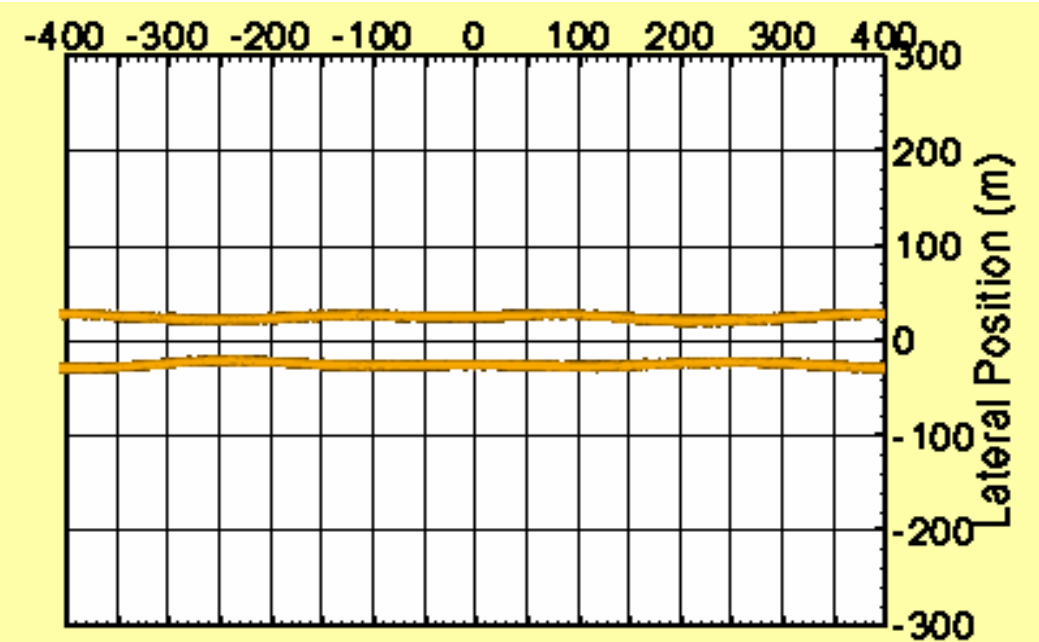

Figure 6. Top view of wake vortex during phase-1 $(T=3)$ for $N^{*}=0, \varepsilon^{*}=0.03$ case.

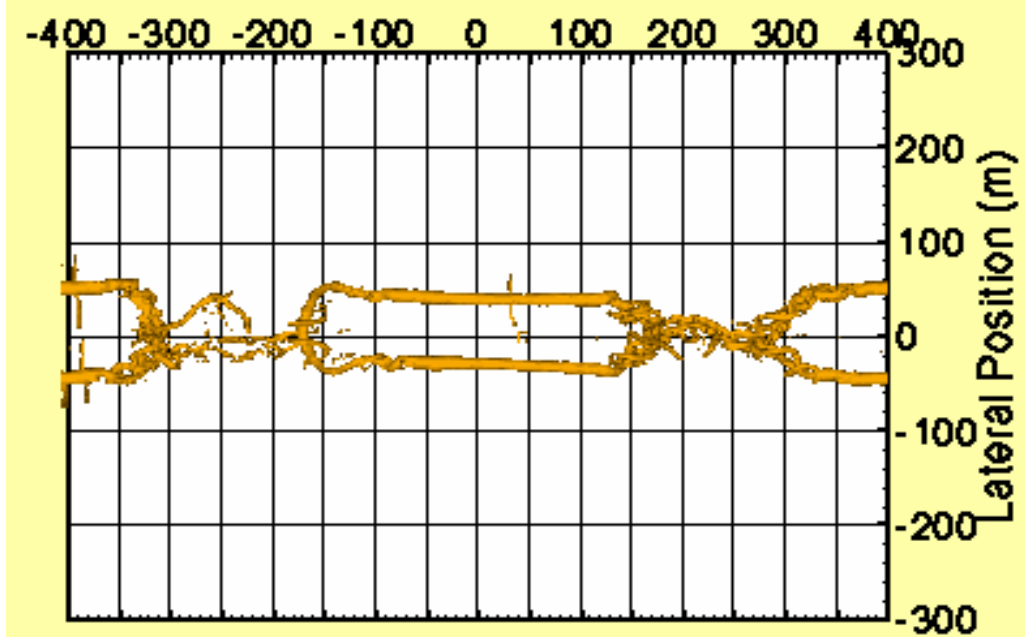

Figure 7. Same as Figure. 6 , but during time of phase-2 decay $(T=6)$.

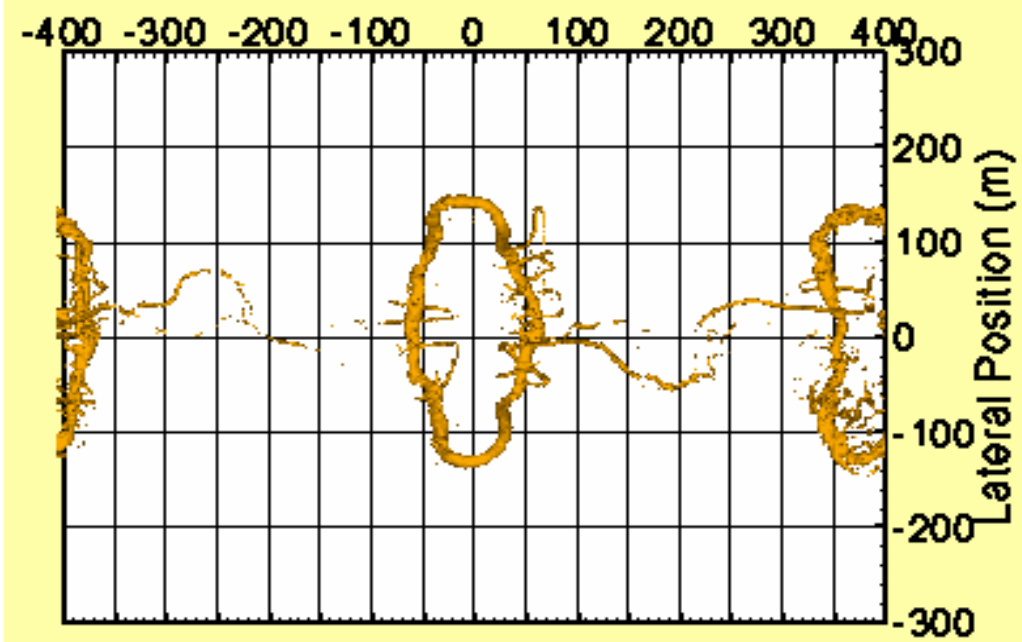

Figure 8. Same as Figure 6, but during phase-3 decay $(T=9)$. 


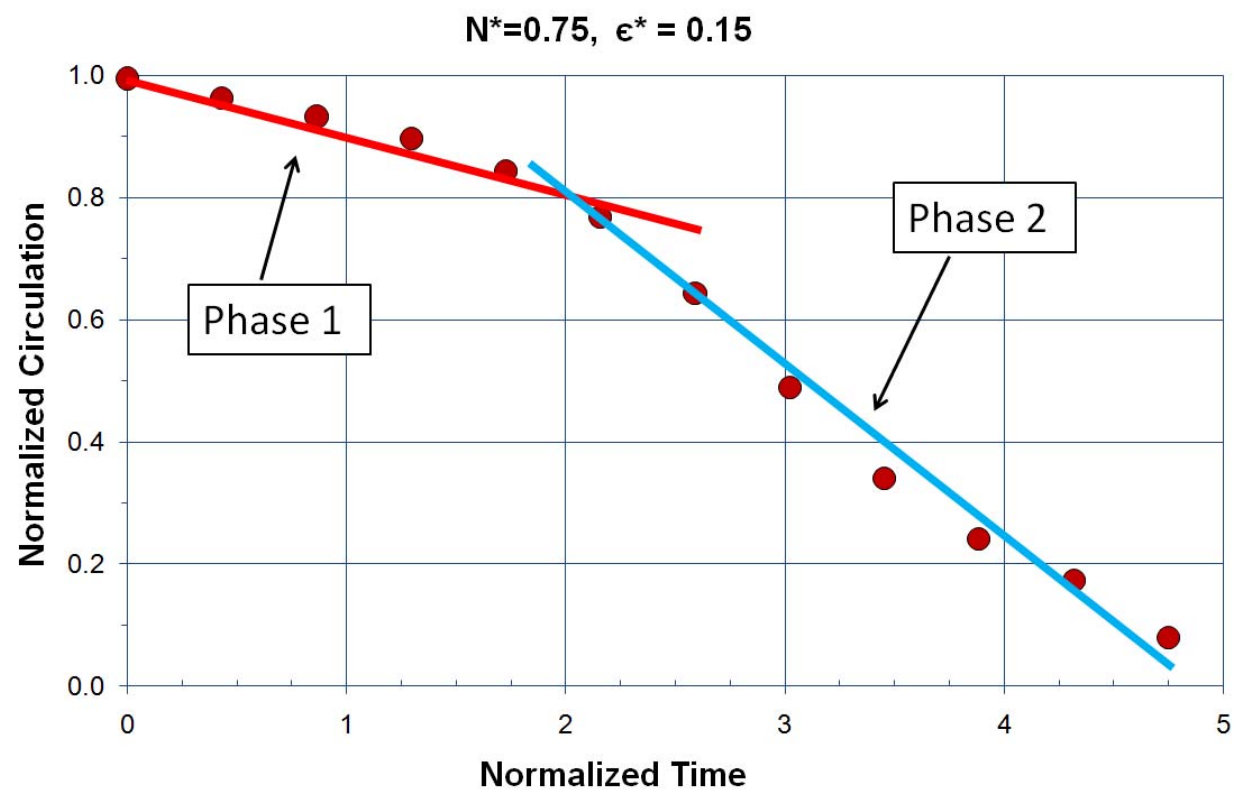

Figure 9. As in Figure 5b, but for a stable atmosphere $\left(N^{*}=0.75\right)$.

For the simulations with greater levels of atmospheric turbulence or stable stratification, the ring vortices decay more quickly and only one or two phases of decay may be obvious. For example, in a stratified atmosphere the third phase may all but disappear (Figure 9); while in the case of high turbulence, the rate of decay is rapid and nearly linear (Figure 10).

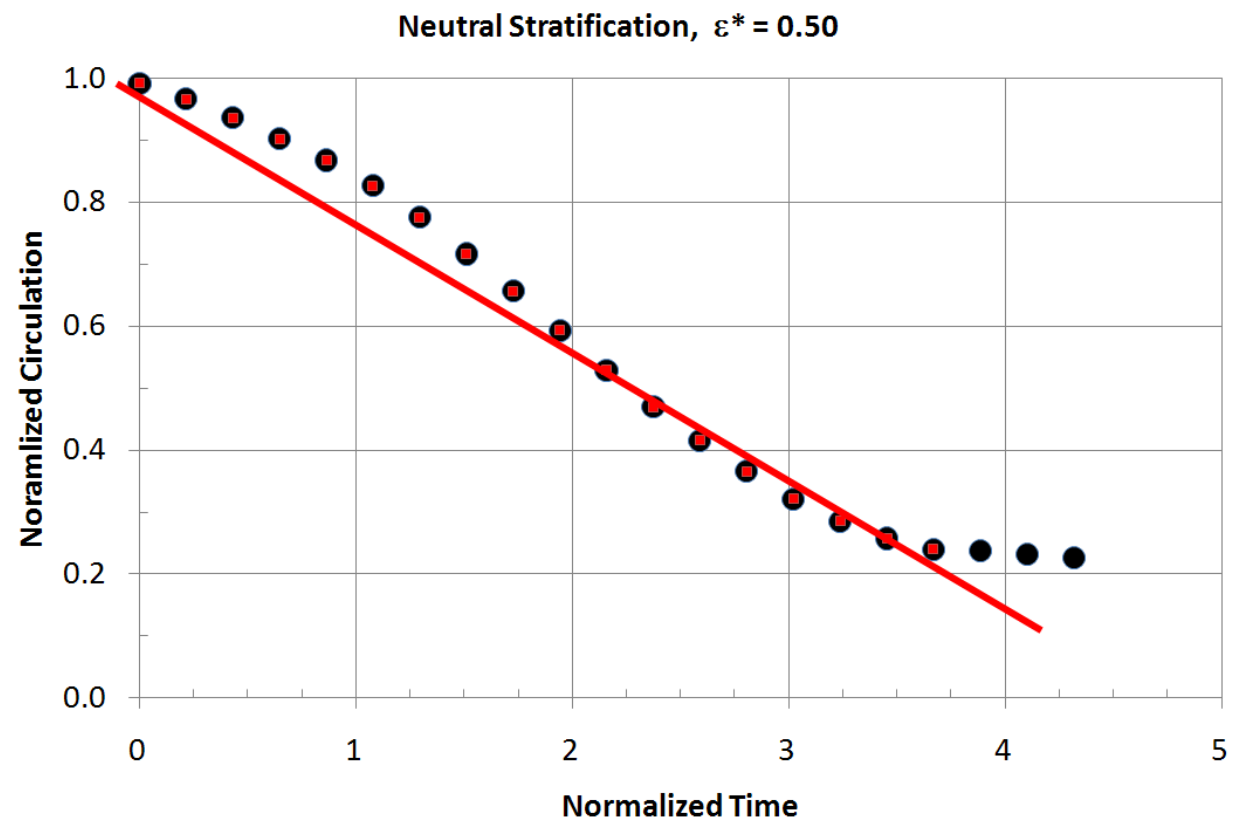

Figure 10. Nearly linear circulation decay for strong turbulence $\left(\varepsilon^{*}=0.5\right)$ in a neutral atmosphere. 


\section{Summary and Conclusion}

A parametric set of LES simulations were conducted to better understand the influence of turbulence and stratification on wake decay, and to provide guidance for future improvements of existing wake prediction models. This study shows that the linking time of wake vortices depends upon atmospheric turbulence intensity and can be predicted with Sarpkaya's analytical formulation. Sensitivity of the linking time to environmental stratification is very slight. Although, in some cases, strong stratification can result in demise of a vortex before linking can occur.

Our results show important characteristics of wake decay, especially during the ring vortex stage. Wake vortices that are embedded within environments having low-turbulence and near-neutral stratification, undergo three phases of vortex decay. The second phase, which is associated with vortex linking, has a steeper rate of decay than the first phase. A third phase, with a more gradual rate of decay, occurs in association with the formation of ring vortices.

Current wake vortex fast-time prediction models, at most, have two phases of decay. In future work, the TDAWP model can be modified to incorporate finding from this study.

\section{Acknowledgments}

This work is sponsored under NASA's Concepts \& Technology Development Project of the Airspace Systems Program. The numerical simulations were conducted using the NASA Pleiades supercomputer cluster.

\section{Appendix: Effect of Reynolds Averaging of Governing Equations on Spectra}

The values predicted at each grid point from a large eddy simulation do not represent point values, but represent volumetric averages. Therefore, variance or energy spectra computed from this data will not maintain a constant slope at the highest resolved wavenumbers. To understand how Reynolds filtering of the governing equation set may affect the solution, we perform the following analysis that is generalized from Pasquill and Smith ${ }^{28}$ and Deardorff. $^{29}$

Assume any variable, $u$, is composed of sinusoidal components:

$$
u=a \sin k x
$$

where, $x$ is the coordinate in space, and $k$ is the wavenumber.

Then assume a filter width of $n \Delta x$

$$
\bar{u}=\frac{1}{n \Delta x} \int_{x^{\prime}-n \Delta x / 2}^{x^{\prime}+n \Delta x / 2} u d x
$$

or,

$$
\bar{u}=\frac{2 a}{k n \Delta x} \sin (k n \Delta x / 2) \sin \left(k x^{\prime}\right)
$$

The amplification factor, $p$, which is a function of wavenumber is:

$$
p(k)=\left(\frac{\bar{u}}{u}\right)^{2}=\left(\frac{\sin (k n \Delta x / 2)}{k n \Delta x / 2}\right)^{2}
$$

where $n=1$ for a filter width of $\Delta x$ and $n=2$ for a filter width of $2 \Delta x$. The subgrid Reynolds averaging in TASS assumes a $2 \Delta x$ filter width, so $n=2$ is appropriate. The amplification factor indicates how much the spectrum should be amplified as a function of wavenumber to compensate for the Reynold's averaging of the governing equations. 


\section{References}

${ }^{1}$ Crow, S. C., "Stability Theory for a Pair of Trailing Vortices," AIAA Journal, Vol. 8, No. 12, December 1970, pp. 2172-2179.

${ }^{2}$ Crow, S.C., and Bate, E.R., "Lifespan of Trailing Vortices in a Turbulent Atmosphere," J. Aircraft, Vol. 13, No. 7, July 1976, pp. 476-482.

${ }^{3}$ Sarpkaya, T., and Daly, J.J., "Effect of Ambient Turbulence on Trailing Vortices," J. Aircraft, Vol. 24, No. 6, 1987, pp. 399-404.

${ }^{4}$ Spalart, P.R., “Airplane Trailing Vortices,” Ann. Rev. Fluid Mech., Vol. 30, 1998, pp. 107-138.

${ }^{5}$ Holzapfel, F., Gerz, T., and Baumann, R., "The Turbulent Decay of Trailing Vortex Pairs in Stably Stratified Environments," Aerosp. Sci. Technol., Vol. 5, 2001, pp. 95-108.

${ }^{6}$ Holzapfel, F., "Probabilistic Two-Phase Wake Vortex Decay and Transport Model," J. Aircraft, Vol. 40, MarchApril 2003, pp. 323-331.

${ }^{7}$ Proctor, F.H., and Switzer, G.F., "Numerical Simulation of Aircraft Trailing Vortices," 9th Conf. on Aviation, Range and Aerospace Meteorology, American Meteorology Society, September 2000, pp. 511-516.

${ }^{8}$ Proctor, F.H., Hamilton, D.W., and Switzer, G.F. “TASS Driven Algorithms for Wake Prediction,” January 2006, AIAA 2006-1073.

${ }^{9}$ Proctor, F.H., "Evaluation of Fast-Time Wake Vortex Prediction Models," January 2009, AIAA 2009-0344.

${ }^{10}$ Greene, G. C., “An Approximate Model of Vortex Decay in the Atmosphere,” Journal of Aircraft, Vol. 23, No. 7, July 1986, pp. 566-573.

${ }^{11}$ Sarpkaya, T., Robins, R.E., and Delisi, D.P., "Wake-Vortex Eddy-Dissipation Model Predictions Compared with Observations," Journal of Aircraft, Vol. 38, No. 4, July-August 2001, pp. 687- 692.

${ }^{12}$ Robins, R.E., Delisi, D.P., and Greene, G.C., “Algorithm for Prediction of Trailing Vortex Evolution,” J. Aircraft, Vol. 38, No. 5, September-October 2001, pp. 911-917.

${ }^{13}$ Proctor, F.H., "The Terminal Area Simulation System, Volume 1: Theoretical Formulation," April 1987, NASA CR-4046.

${ }^{14}$ Switzer, G.F., and Proctor, F.H., "Numerical Study of Wake Vortex Behavior in Turbulent Domains with Ambient Stratification," January 2000, AIAA-2000-0755.

${ }^{15}$ Han, J., Lin, Y. -L., Schowalter, D. G., Arya, S. P., and Proctor, F. H., "Large Eddy Simulation of Aircraft Wake Vortices within Homogeneous Turbulence: Crow Instability," AIAA Journal, Vol. 38, No. 2, February 2000, pp. 292-300.

${ }^{16}$ Proctor, F.H., "Interaction of Aircraft Wakes from Laterally Spaced Aircraft," January 2009, AIAA 2009-0343.

${ }^{17}$ Switzer, G.F., "Validation Tests of TASS for Application to 3-D Vortex Simulations," October 1996, NASA CR4756.

${ }^{18}$ Proctor, F. H., "The NASA-Langley Wake Vortex Modeling Effort in Support of an Operational Aircraft Spacing System,” January 1998, AIAA 98-0589.

${ }^{19}$ Lamb, H., Hydrodynamics, 6th Ed., Cambridge University Press, 1932, 738 pp. 
${ }^{20}$ Vincent, A. and Meneguzzi, M., "The Spatial Structure and Statistical Properties of Homogeneous Turbulence," Journal of Fluid Mechanics, Vol. 225, 1991, pp. 1-20.

${ }^{21}$ Han, J., Lin, Y.-L., Arya, S.P., and Proctor, F.H., "Numerical Study of Wake Vortex Decay and Descent within Homogeneous Turbulence," AIAA Journal, Vol. 38., No. 4, April 2000, pp. 643-656.

${ }^{22}$ Kolmogorov, A.N., "The Local Structure of Turbulence in Incompressible Viscous Fluid for Very Large Reynolds Number," Dokl. Akad. Nauk SSSR, Vol. 30, 1941, pp. 9-13.

${ }^{23}$ Hinton, D.A., and Tatnall, C.R., "A Candidate Wake Vortex Strength Definition for Application to the NASA Aircraft Vortex Spacing System (AVOSS)," 1997, NASA TM-110343.

${ }^{24}$ Sarpkaya, T., "New Model for Vortex Decay in the Atmosphere," AIAA Journal, Vol. 37, No. 1, January 2000, pp. 53-61.

${ }^{25}$ Leweke, T., and Williamson, C.H.K, “Cooperative Elliptic Instability of a Vortex Pair,” J. Fluid Mech., Vol. 360, 1998, pp. 85-119.

${ }^{26}$ Delisi, D.P., and Robins, R.E., "Short-scale Instabilities in Trailing Wake Vortices in a Stratified Fluid," AIAA Journal, Vol. 38, 2000, pp. 1916-1923.

${ }^{27}$ Loucel, R.E., and Crouch, J.D., "Flight-Simulator Study of Airplane Encounters with Perturbed Trailling Vortices," Journal of Aircraft, Vol. 42, No.4, July-August 2005, pp. 924-931.

${ }^{28}$ Pasquill, F., and Smith, F.B., Atmospheric Diffusion, $3^{\text {rd }}$ edition, Ellis Horwood Limited, 1983, 437 pp.

${ }^{29}$ Deardorff, J.W., "On the Magnitude of the Subgrid Scale Eddy Coefficient," Journal of Computational Physics, Vol. 7, 1971, pp. 120-133. 